\title{
ASYMPTOTIC BEHAVIOUR AND BLOW-UP OF SOME UNBOUNDED SOLUTIONS FOR A SEMILINEAR HEAT EQUATION
}

\author{
by D. E. TZANETIS
}

(Received 26th May 1994)

\begin{abstract}
The initial-boundary value problem for the nonlinear heat equation $u_{t}=\Delta u+\lambda f(u)$ might possibly have global classical unbounded solutions, $u^{*}=u\left(x, t ; u_{0}^{*}\right)$, for some "critical" initial data $u_{0}^{*}$. The asymptotic behaviour of such solutions is studied, when there exists a unique bounded steady state $w(x ; \lambda)$ for some values of $\lambda$. We find, for radial symmetric solutions, that $u^{*}(r, t) \rightarrow w(r)$ for any $0<r \leq 1$ but $\operatorname{supu}^{*}(\cdot, t)=u^{*}(0, t) \rightarrow \infty$, as $t \rightarrow \infty$. Furthermore, if $\hat{u}_{0}>u_{0}^{*}$, where $u_{0}^{*}$ is some such critical initial data, then $\hat{u}=u\left(x, t ; \hat{u}_{0}\right)$ blows up in finite time provided that $f$ grows sufficiently fast.
\end{abstract}

1991 Mathematics subject classification: $35 \mathrm{~K} 57$

\section{Introduction}

We consider the initial-boundary value problem for a semilinear heat equation of the form:

$$
\begin{gathered}
u_{t}=\Delta u+\lambda f(u), x \text { in } \Omega, t>0 \\
u(x, t)=0, x \text { on } \partial \Omega, t>0 \\
u(x, 0)=u_{0}(x), x \text { in } \Omega
\end{gathered}
$$

which models the temperature distribution of a large number of physical phenomena from physics, chemistry and biology. The term $\lambda f(u)$ represents nonlinear heat generation. Here $\lambda$ is a positive parameter, $f$ is positive, increasing and convex, i.e.

$$
f(s)>0 \text { for } s \geq 0, f^{\prime}(s)>0, f^{\prime \prime}(s)>0 \text { for } s>0
$$

and its growth at infinity is such that

$$
\int_{0}^{\infty} \mathrm{ds} / f(s)<\infty
$$

The initial condition $u_{0}(x)$ is a positive function in $L^{\infty}(\Omega)$ and satisfies the compatibility 

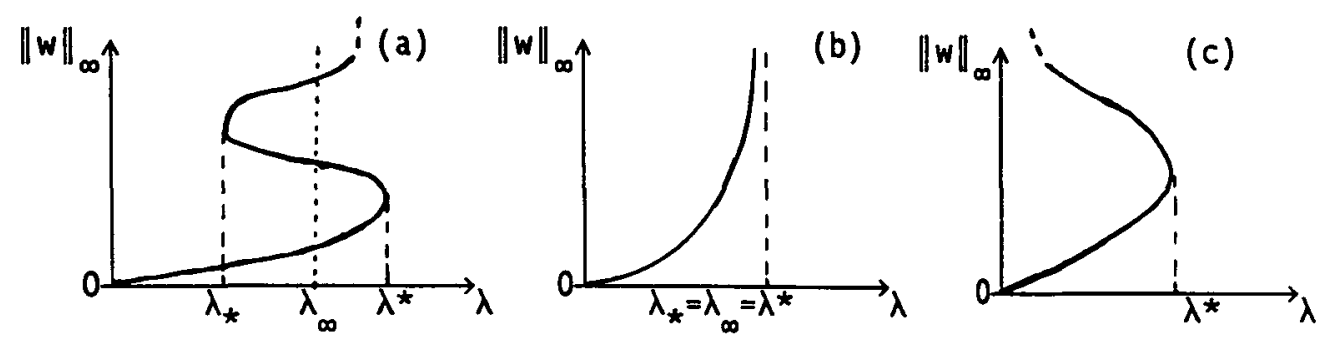

FIGURE 1. Response diagrams for the problem (1.4)

condition $u_{0}(x)=0$ on $\partial \Omega$, i.e. $u_{0} \in X=\left\{u_{0}(x) \in L^{\infty}(\Omega): u_{0}(x)>0\right.$ in $\Omega, u_{0}(x)=0$ on $\left.\partial \Omega\right\}$. We also take $\Omega$ to be a bounded domain of $\mathbb{R}^{N}(N \geq 2)$ with a $C^{2+a}$ boundary $\partial \Omega$, $0<a<1$.

A steady state corresponding to $(1.1 \mathrm{a}, \mathrm{b})$ satisfies:

$$
\begin{gathered}
\Delta w+\lambda f(w)=0, x \text { in } \Omega \\
w(x)=0, x \text { on } \partial \Omega .
\end{gathered}
$$

It is well known that the problem (1.4) may or may not have a solution depending on the values of $\lambda[1,7]$. Figure 1 shows possible forms of the response diagram for the problem (1.4) $[1,6]$.

In any case there is a $\lambda^{*}$ such that (1.4) has a solution for $\lambda<\lambda^{*}$ but not for $\lambda>\lambda^{*}$. We can observe from figure $1(a, b)$ that in these cases there is additionally a $\lambda *$ such that for $0<\lambda<\lambda_{*}$ the Problem (1.4) has a unique solution $[1,6,7]$.

It is also known that for $\lambda>\lambda^{*}, u(x, t ; \lambda)$, the solution to (1.1), blows up in finite time, i.e. there exists a $t^{*}<\infty$ such that

$$
\limsup _{t \rightarrow t^{*}-}\|u(\cdot, t)\|_{\infty}=\infty,\|(\cdot)\|_{\infty}=\sup (\cdot)
$$

For $\lambda<\lambda^{*}$ there is at least a minimal steady stage $w_{m}$ which is asymptotically stable $[1,4,16]$, while for $\lambda_{*}<\lambda<\lambda^{*}$ there is at least a second, non-minimal, solution $w$ of $(1.4)$, which plays the role of "critical" initial data, i.e. if $u_{0}<w$ then $u\left(\cdot, t ; u_{0}\right) \rightarrow w_{m}(\cdot)$ as $t \rightarrow \infty$,

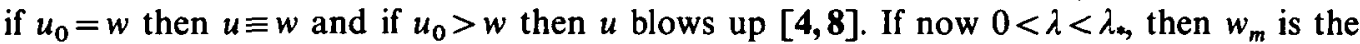
unique solution to (1.4) and behaves as an attractor, i.e. there is a domain, or region, of attraction, which we denote by $A\left(w_{m}\right)$, such that if $u_{0} \in A\left(w_{m}\right)$ then $u\left(\cdot, t ; u_{0}\right) \rightarrow w_{m}$ as $t \rightarrow \infty$. In this case the non-minimal $w$ is replaced by some other type of critical initial data $u_{0}^{*}$, certainly in the sense that if $u_{0}<u_{0}^{*}$ then $u\left(x, t ; u_{0}\right)$ is bounded and $u \rightarrow w_{m}$ uniformly as $t \rightarrow \infty$, while if $u_{0} \gg u_{0}^{*}$ then $u$ blows up [8]: $u_{0}^{*} \in \partial_{+} A\left(w_{m}\right)$. With these critical data $u_{0}^{*}, u^{*}=u\left(x, t, u_{0}^{*}\right)$ is a weak solution ( $L^{1}$-solution) to (1.1) for all time, unbounded in $L^{\infty}$ but bounded in $L^{1}[10,13]$.

We still do not have a complete answer regarding the global existence of unbounded classical solutions $u^{*}$ to (1.1). For $N=3$ and $f(u)=e^{u}$ some solutions $u^{*}$ can blow up in a 
self-similar form at a time $t^{*}<\infty$. These can continue to exist after $t^{*}$ as bounded solutions [10]. We characterise this behaviour as partial blow-up (when the solution continues to exist beyond $t^{*}$ as a weak solution) in contrast with complete blow-up $\left(u=\infty\right.$ for $\left.t>t^{*}\right)$ [10]. It is not clear if there exist such solutions for other problems of the type (1.1) when the response diagram is of form 1(a) or 1(b), for instance when $f(u)=e^{u}, N \geq 10$, since here the method of construction of self-similar solutions fails. It is however possible to find more general problems where partial blow-up takes place in a manner other than self-similar (see Section 5 in [10]).

We speculate that there are cases where $u^{*}$ is global classical unbounded solution, not only at $\lambda=\lambda^{*}, f(u)=e^{u}, N \geq 10$, [9], but also for $0<\lambda<\lambda$. for some $N>2$. (We recall that in [9], for $\lambda=\lambda^{*}$, a solution $u^{*}$ (under certain initial conditions) is found to exist for all time and to be unbounded. Moreover $u^{*} \rightarrow w^{*}$ a.e. as $t \rightarrow \infty$, where $w^{*}$ is a singular steady state.)

In this paper we analyse the asymptotic behaviour of any such solution $u^{*}$. Also we discuss what happens to the solution of (1.1) if the initial data are greater than critical $u_{0}^{*}$.

In Section 2 , for the radially symmetric case, we prove that, given suitable restrictions on the initial data, $u^{*}(r, t) \rightarrow w_{m}(r), 0<r \leq 1$ as $t \rightarrow \infty$. In the following section we consider $\hat{u}=u\left(x, t ; \hat{u}_{0}\right)$ with $\hat{u}_{0}>u_{0}^{*}$, and find that $\hat{u}$ blows up in finite time, provided that $f(s)$ grows fast enough.

\section{Convergence of unbounded solutions}

We study the asymptotic behaviour of global classical unbounded solutions to (1.1) when $\Omega$ is the unit ball, i.e. $\Omega=B(0,1)$, in $\mathbb{R}^{N}$ for some $N>1$ (for $N=1, \lambda_{*}=0$ ). Thus (1.1) can be written as

$$
\begin{gathered}
u_{t}^{*}=\Delta_{r} u^{*}+\lambda f\left(u^{*}\right), 0<r<1, t>0 \\
\frac{\partial u^{*}(0, t)}{\partial r}=u^{*}(1, t)=0, t>0 \\
u^{*}(r, 0)=u_{0}^{*}(r), 0<r<1 .
\end{gathered}
$$

The corresponding steady state problem of $(2.1 \mathrm{a}, \mathrm{b})$ is:

$$
\begin{gathered}
\Delta_{r} w+\lambda f(w)=0,0<r<1 \\
w^{\prime}(0)=0, w(1)=0 .
\end{gathered}
$$

Here $\Delta_{r}(\cdot)=\partial^{2}(\cdot) / \partial r^{2}+((N-1) / r) \partial(\cdot) / \partial r$.

The conditions $\partial u^{*}(0, t) / \partial r=w^{\prime}(0)=0$ are a consequence of boundedness of solutions

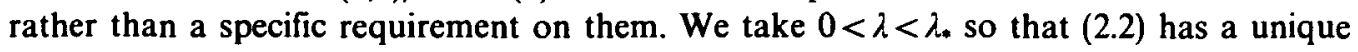
solution $w_{m}$ which, in the following, we denote by $w$.

We now suppose that the following hypothesis is fulfilled. 
$\left(H_{1}\right)$ There are "critical" initial data $u_{0}^{*}$ such that $u^{*}=u\left(x, t ; u_{0}^{*}\right)$ is a global unbounded classical solution to (1.1) or (2.1).

These initial data $u_{0}^{*}$ are critical in the sense that for $u_{0}<u_{0}^{*} u\left(x, t ; u_{0}\right)$ is uniformly bounded, whereas for $\hat{u}_{0} \geq u_{0}^{*}, \hat{u}=u\left(x, t ; \hat{u}_{0}\right)$ is unbounded in $L^{\infty}$-norm, i.e. $u_{0}^{*} \in \partial_{+} A\left(w_{m}\right)$ [10]. In addition by standard arguments $u\left(\cdot, t ; u_{0}\right) \rightarrow w(\cdot)$ uniformly, see [13] and the references therein.

The hypotheses $\left(H_{1}\right)$ implies that there exists a sequence of times $\left(t_{n}\right)$ such that sup $u^{*}\left(\cdot, t_{n}\right) \rightarrow \infty, t_{n} \rightarrow \infty$ as $n \rightarrow \infty$. Moreover we can show that $\sup u^{*}(\cdot, t) \rightarrow \infty$ as $t \rightarrow \infty$. Let us assume for a contradiction, that there exists a sequence $t_{n} \rightarrow \infty$ with $\left\|u^{*}\left(\cdot, t_{n}\right)\right\|<k$; then we may deduce the existence of an omega limit set of $u^{*}: \omega\left(u_{0}^{*}\right)=\left\{v \in L^{\infty}(\Omega): \exists T_{n}\right.$ $\rightarrow \infty$ s.t. $\left.u\left(\cdot, T_{n} ; u_{0}^{*}\right) \rightarrow v\right\} \neq \varnothing$, then by standard arguments $\omega\left(u_{0}^{*}\right)=\{w\}$, since $w$ is the unique steady state. We deduce that $u^{*} \rightarrow w$, contradicting the unboundedness of $u^{*}$, see $[12,13]$.

Also following Lacey and Tzanetis [10], see also [13], we may deduce that $u^{*}$ is an $L^{1}$-solution i.e.

$$
\begin{gathered}
u^{*} \in C\left([0, T] ; L^{1}(\Omega)\right), \\
f\left(u^{*}\right) \in L^{1}\left(\Omega_{\tau}\right), \Omega_{T}=\Omega \times(0, T)
\end{gathered}
$$

and $u^{*}$ satisfies

$$
\int_{\mathbf{\Omega}}[u \psi]_{t_{0}}^{t_{0}} d x-\int_{t_{0}}^{t} \int_{\mathbf{\Omega}} u \psi_{t} d x d s=\int_{t_{0}}^{t} \int_{\Omega}(u \Delta \psi+\lambda \psi f(u)) d x d s
$$

for every $\psi \in C^{2}\left(\bar{\Omega}_{T}\right)$ with $\psi=0$ on $\partial \Omega$ and $0 \leq t_{0}<t \leq T$ for every large $T$. Furthermore the following estimate holds:

$$
\int_{\mathbf{\Omega}} u^{*}(x, t) d x<c, c \text { a constant }
$$

It is obvious that (2.4) excludes the possibility of complete blow-up occurring for $u^{*}$, while $\left(H_{1}\right)$ does not permit partial blow-up to take place.

We also impose the following hypothesis:

$\left(H_{2}\right)$ There is no non-negative singular solution to (1.4) or (2.2) for $\lambda \in\left(0, \lambda_{*}\right)$.

Note that $\left(\mathrm{H}_{2}\right)$ is known to hold in certain cases, for instance when $f(s)=s^{p}$ (unforced case $f(0)=0$ ), see [15]. In the forced case $(f(0)>0)$, which is our interest, we can show, by using phase plane analysis, that $\left(\mathrm{H}_{2}\right)$ is true for the Problem (2.2) at least when $f(s)=e^{s}$ or $f(s)=(1+s)^{p}, p>0$ and $N \geq 3$.

Indeed, by making the transformation 
where

$$
r=e^{s_{0}-s}, w(r)=M+z(s)+2 s
$$

$$
M=w(0) \text { and } z\left(s_{0}\right)=-M-2 s_{0}, 2 s_{0}=\ln \frac{2(N-2)}{\lambda e^{M}}
$$

the Problem (2.2) for $f(s)=e^{s}$ takes the form:

By setting

$$
\begin{gathered}
\frac{1}{N-2} \ddot{z}-\dot{z}-2+2 e^{2}=0, s_{0}<s<\infty \\
z(\infty)=-\infty, \dot{z}(\infty)=-2 .
\end{gathered}
$$

$$
y(s)=\dot{z}(s)+2, x(s)=2(N-2) e^{z(s)},
$$

on the $(x, y)$-phase plane, $(2.6)$ becomes:

$$
\begin{gathered}
\dot{y}=(N-2) y-x, s_{0}<s<\infty \\
\dot{x}=x(y-2), s_{0}<s<\infty \\
x(\infty)=y(\infty)=0
\end{gathered}
$$

with compatibility condition,

$$
2 s_{0}=\ln \frac{2(N-2)}{x\left(s_{0}\right) e^{M}}, \lambda=x\left(s_{0}\right) \text { and } a=-w(1)=y\left(s_{0}\right) .
$$

The system (2.7) has two critical points, at $(0,0)$ and $(2(N-2), 2)$. Then it can be proved that there exists a unique heteroclinic orbit joining the point $(0,0)$ and $\left(x\left(s_{0}\right), y\left(s_{0}\right)\right)=$ $(\lambda, a)$. Taking $s_{0} \rightarrow-\infty$ and studying the behaviour of the solution to (2.7), subject to (2.8), one can show that as $s_{0} \rightarrow-\infty$ either $\left(x\left(s_{0}\right), y\left(s_{0}\right)\right) \rightarrow(2(N-2), 2)$ or $\left(x\left(s_{0}\right), y\left(s_{0}\right)\right)$ spirals about $(2(N-2), 2)$. More precisely, for each pair $\left(x\left(s_{0}\right), y\left(s_{0}\right)\right)$, which is equal to $(\lambda, a)$ via the compatibility condition (2.8) and the change of variables (2.5), we obtain

$$
w(r)=M+2 s+\ln \left(\frac{x(s)}{2(N-2)}\right), r(s)=e^{s_{0}-s}
$$

The above observations are depicted by the response diagrams shown in fig. 2 .

The critical point $\left(\lambda_{\infty}, 2\right)$ corresponds to the singular solution $w_{\infty}>0$ which satisfies (2.2. a,c) $w_{\infty}(r) \rightarrow \infty$ as $r \rightarrow 0$ and is a limit of regular solutions of $(2.2)[6,9]$. From the above response diagrams it is apparent that there is no singular solution to (2.2) for $0<\lambda<\lambda_{e}, f(s)=e^{3}$. Thus $\left(H_{2}\right)$ is fulfilled. Also these diagrams have some similar features to figure 1 (a) and (b) respectively. For more details see Bebernes and Eberly [2], as well as Joseph and Lundgren [6], where in addition the case of $f(s)=(1+s)^{p}$ is studied. 


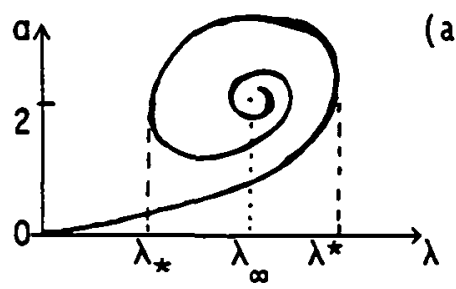

(a) $3 \leq N \leq 9, \lambda_{*}<\lambda_{\infty}=2(N-2)<\lambda^{*}$ (a)

$$
a=-w^{\prime}(1)
$$

(b)

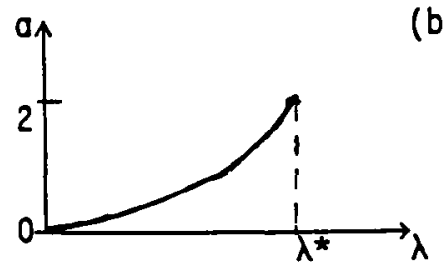

(b) $N \geq 10, \lambda_{*}=\lambda_{\infty}=2(N-2)=\lambda^{*}$

FIGURE 2. The response diagrams for the problem (2.2)

We note that any solution $w$ of (1.4) is positive and if $\Omega$ is a ball then $w$ is radially symmetric and radially decreasing i.e. $w(x)=w(r)>0, w^{\prime}(r)<0,0<r<1$, and $w$ satisfies (2.2). Also any solution $u$ of (1.1) is positive and if again $\Omega$ is a ball with $u_{0}(x)=u_{0}(r)$ then $u(x, t)=u(r, t)$ is radially symmetric and satisfies (2.1). Furthermore if $u_{0}^{\prime}(r)<0$ then $u_{r}(r, t)<0,0<r<1,0<t<T$ i.e. $u$ is radially decreasing, see Gidas et al. [5].

Our purpose is to show that $u^{*}(r, t)$, the solution to $(2.1)$, is eventually decreasing in $r$; this will be crucial to what follows. If we had that $u_{0}^{*}(r)<0$ then by applying the maximum-minimum principle and Hopf's boundary point lemma we would obtain that $u_{r}^{*}(r, t)<0,0<r<1,0<t<T$, for every $T$. Here we do not require $u_{0}^{* \prime}(r)<0$ however we can still proceed by using a result due to $\mathrm{Ni}$ and Sacks [14]. The following lemma holds.

Lemma 2.1. Let $u^{*}(r, t)$ be the solution to (2.1) and $\left(H_{1}\right),(1.2),(1.3)$ be satisfied; then there exists $a 0<t_{0}<\infty$ such that $u_{r}(r, t)<0,0<r \leq 1, t \geq t_{0}$.

Analogous to the blow-up point we may define $x \in \bar{\Omega}$ to be a divergence point of $u$ if there exists a sequence $\left(x_{m}, t_{m}\right)$ such that $x_{m} \rightarrow x, t_{m} \rightarrow \infty$ and $u\left(x_{m}, t_{m}\right) \rightarrow \infty$ as $m \rightarrow \infty$. Then we have the following theorem.

Theorem 2.2. Let $u^{*}$ be the solution to (2.1) and $\left(H_{1}\right),(1.2),(1.3)$ be satisfied, then the measure of the set of divergence points of $u^{*}$ is zero. Furthermore, $r=0$ is the only divergence point of $u^{*}$, and for any $r \in(0,1], u^{*}(r, t)$ is bounded for every $t>0$.

Proof. Let us assume for a contradiction that there exists some $r_{0}>0$ such that $u^{*}\left(r_{m}, t_{m}\right) \rightarrow \infty$ as $t_{m} \rightarrow \infty$ and $r_{m} \rightarrow r_{0}$. Lemma 2.1 implies $u^{*}\left(r, t_{m}\right) \rightarrow \infty$ as $t_{m} \rightarrow \infty$ uniformly on $\left[0, r_{0} / 2\right]$. But this result contradicts (2.4), i.e. that $\int_{\Omega} u^{*} d x$ is bounded as $t \rightarrow \infty$. Hence the only divergence point can be $r=0$. Since $u^{*}$ is not uniformly bounded, $r=0$ is indeed a divergence point. For every $0<r \leq 1, u^{*}(r, t)$ is bounded for $t>0$, while from the result that sup $u^{*} \rightarrow \infty$ above, $u^{*}(0, t)=\sup u^{*}(\cdot, t) \rightarrow \infty$ as $t \rightarrow \infty$.

We now define a ring-shaped domain $\Omega_{\eta} \subset \Omega$ for a given $\eta \in(0,1)$ by

$$
\Omega_{\eta}=\{x \in \Omega: \eta<|x|<1\}
$$


There holds the following:

Proposition 2.3. Let us assume that $\left(H_{1}\right)$ holds and $u^{*}$ satisfies (2.1). Then

$$
\int_{\Omega_{\eta}} u_{i}^{*^{2}}(x, t) d x \rightarrow 0 \text { as } t \rightarrow \infty
$$

Proof. We shall first prove that (2.9) holds for a subsequence of time. Indeed, multiplying (2.1a) by $u_{t}^{*}$ and integrating over $\Omega_{\eta} \times\left(t_{0}, T\right), t_{0}>0$, we obtain

$$
\begin{gathered}
\int_{t_{0}}^{T} \int_{\Omega_{1}} u_{t}^{*^{2}}(x, t) d x d t=\int_{t_{0}} \int_{\Omega_{T}} u_{t}^{*}\left(\Delta u^{*}+\lambda f\left(u^{*}\right)\right) d x d t \\
=\int_{t_{0}}^{T} \int_{\partial \Omega_{\eta}} u_{i}^{*} \frac{\partial u^{*}}{\partial n} d x d t+\int_{t_{0}}^{T} \int_{\Omega_{1}}\left(-\frac{1}{2} \frac{\partial}{\partial t}\left|\nabla u^{*}\right|^{2}+\lambda \frac{d}{d t} F\left(u^{*}\right)\right) d x d t \\
\leq-\int_{t_{0}}^{T} \int_{|x|=\eta} u_{t}^{*} \frac{\partial u^{*}}{\partial n} d x d t+\frac{1}{2} \int_{\Omega_{i}}\left|\nabla u^{*}\left(x, t_{0}\right)\right|^{2} d x+\lambda \int_{\Omega_{1}} F\left(u^{*}(x, T)\right) d x,
\end{gathered}
$$

where we have used $F(u)=\int_{0}^{u} f(s) d s$ and

$$
\int_{t_{0}}^{T} \int_{|x|=1} u_{t}^{*} \frac{\partial u^{*}}{\partial n} d x d t=0 .
$$

Theorem 2.2 implies that $u^{*}(x, t)$ is uniformly bounded in $\Omega_{\eta / 2} \times[0, \infty)$ so that by using standard parabolic regularity theory, see Ladyzenskaja et al. [11], we obtain that $u^{*}, u_{t}^{*}, u_{x_{i}}^{*}, u_{x_{i x_{j}}}^{*}$ are bounded in $C^{a, a / 2}\left(\bar{\Omega}_{\eta} \times\left[t_{0}, \infty\right)\right)$ for some $t_{0}>0,0<a<1$, where $C^{a, a / 2}$ represents the space of Hölder continuous functions with exponent $a$ for $x$, and $a / 2$ for $t$. From this observation we can bound the right-hand side of (2.10) from above by a constant, independent of $T$. Hence we have

$$
\int_{t_{0}}^{T} \int_{\Omega_{n}} u_{t}^{*^{2}}(x, t) d x d t<c
$$

and since $c$ is independent of $T$

$$
\int_{t_{0}}^{\infty} \int_{\Omega_{1}} u_{t^{*}}^{*^{2}}(x, t) d x d t<c
$$

The last relation implies that there exists a subsequence of times $\left(t_{k}\right)$ such that

$$
\int_{\Omega_{1}} u_{t}^{*^{2}}\left(x, t_{k}\right) d x \rightarrow 0 \text { as } t_{k} \rightarrow \infty
$$


To see that (2.12) holds for all subsequences of time, we appeal again to the fact that $u_{i}^{*}$ is bounded in $C^{a, a / 2}\left(\bar{\Omega}_{\eta} \times\left[t_{0}, \infty\right)\right)$. This implies that

$$
\int_{\Omega_{n}} u_{t}^{*^{2}}(x, t) d x=v(t)
$$

is uniformly continuous on $\left[t_{0}, \infty\right)$. Let us assume now that there exists a subsequence $t_{m} \rightarrow \infty$ such that $v\left(t_{m}\right) \nrightarrow 0$ as $t_{m} \rightarrow \infty$; then we can deduce the existence of a constant $c>0$ such that $v\left(t_{m}\right) \geq c>0$ for all $m$, redefining $\left(t_{m}\right)$ if necessary. On the other hand from the uniform continuity of $v(t)$ we can choose some $\delta>0$ such that for a given $\varepsilon \in(0, c / 2)$,

$$
\left|v(t)-v\left(t_{m}\right)\right|<\varepsilon \text { for }\left|t-t_{m}\right|<\delta .
$$

Integrating over $\left(t_{m}-\delta, t_{m}+\delta\right)$ we obtain

and

$$
\int_{t_{m}-\delta}^{t_{m}+\delta} v(t) d t \geq(c-\varepsilon) 2 \delta>c \delta
$$

$$
\int_{t_{0}}^{t_{m}+\delta} v(t) d t \geq \sum_{k=1}^{m} \int_{t_{k}-\delta}^{t_{k}+\delta} v(t) d t \geq m c \delta
$$

From the last relation we deduce

$$
\int_{t_{0}}^{\infty} v(t) d t=\lim _{t_{m} \rightarrow \infty} \int_{t_{0}}^{t_{m}+\delta} v(t) d t \geq \lim _{m \rightarrow \infty} m c \delta=\infty
$$

which contradicts (2.11) (the sequence $\left(t_{m}\right)$ may be chosen so that $t_{m+1}-t_{m} \geq 2 \delta$ ). This completes the proof.

We are now ready to give the main theorem of this section.

Theorem 2.4. Let $u^{*}$ be the solution to (2.1), $0<\lambda<\lambda$. and hypotheses $\left(H_{1}\right),\left(H_{2}\right)$ be satisfied. Then $u^{*}(r, t) \rightarrow w(r)$ as $t \rightarrow \infty$ for any $0<r<1$.

Proof. We first suppose that

$$
u_{r}^{*}(1, t) \rightarrow w^{\prime}(1) \text { as } t \rightarrow \infty
$$

Setting $u^{*}(r, t)=U(r, t)+w(r)$ where $w(r)$ is the solution to (2.2) and substituting into (2.1a) we obtain,

$$
\Delta_{r} U+\lambda[f(U+w)-f(w)]=\Delta_{r} U+\lambda f^{\prime}(y) U=u_{i}^{*}(r, t)
$$

where $w<y<u^{*}$. We now consider the Problem (2.1) as a one-parameter family of 
problems with $t$ as a parameter. The existence of the solution to (2.1) implies that the following family of initial value problems,

$$
\begin{gathered}
U^{\prime \prime}(r ; t)+\frac{N-1}{r} U^{\prime}(r ; t)+\lambda f^{\prime}(y) U(r ; t)=u_{i}^{*}(r, t), 0<r<1, \\
U(1 ; t)=0 \\
U^{\prime}(1 ; t)=u_{r}^{*}(1, t)-w^{\prime}(1)
\end{gathered}
$$

has a solution for each value of the parameter $t>0$. The solution (2.14) can be written in the following integral form:

$$
U(r ; t)==\left[u_{r}^{*}(1, t)-w^{\prime}(1)\right] v(r ; t)+\int_{r}^{1} k(r, s ; t) u_{t}^{*}(s, t) d s
$$

where $v=v(r ; t)$ satisfies

$$
\begin{gathered}
v^{\prime \prime}+\frac{N-1}{r} v^{\prime}+\lambda f^{\prime}(y) v=0,0<r<1 \\
v(1 ; t)=0, v^{\prime}(1 ; t)=1
\end{gathered}
$$

and $k=k(r, s ; t)$ is the solution

$$
\begin{gathered}
k^{\prime \prime}+\frac{N-1}{r} k^{\prime}+\lambda f^{\prime}(y) k=0, r>0, r \neq s \\
k(s, s ; t)=0, k^{\prime}(s, s ; t)=-1,
\end{gathered}
$$

where the derivatives are taken with respect to $r$. From (2.15) we get

$$
|U(r ; t)| \leq\left|u_{r}^{*}(1, t)-w^{\prime}(1)\right||v|+\left|\int_{r}^{1} k(r, s ; t) u_{i}^{*}(s, t) d s\right|
$$

and using Cauchy-Schwarz inequality

$$
\leq\left|u_{r}^{*}(1, t)-w^{\prime}(1)\right||v|+\left(\int_{\eta}^{1} k^{2}(r, s ; t) d s\right)^{1 / 2}\left(\int_{\eta}^{1} u_{t}^{*^{2}}(s, t) d s\right)^{1 / 2} .
$$

As long as $u^{*}(r, t)$ exists and is uniformly bounded in time for any $r \geq \eta>0$, the solution $U(r ; t)$ to (2.14) also exists for any $t>0$ and is bounded independently of $t, 0<\eta \leq r \leq 1$. Consequently $v$, the solution to (2.16), is bounded independently of $t . K(r, s ; t)$, the solution to (2.17), is well defined, and exists for any finite interval $\eta \leq r \leq 1$, is continuous and bounded, and its bound is independent of $t$, and $\int_{\eta}^{1} K^{2} d s<c_{2}$. 
Hence from (2.18) we obtain

$$
|U(r ; t)| \leq\left|u_{r}^{*}(1, t)-w^{\prime}(1)\right| c_{1}+c_{2}\left(\int_{\Omega_{1}} u_{t}^{*^{2}}(x, t) d x\right)^{1 / 2}
$$

where $c_{1}, c_{2}$ depend on $\eta$. Taking the limit as $t \rightarrow \infty$, and from the assumption (2.13) and Proposition 2.3, we deduce that

$$
\left|u^{*}(r, t)-w(r)\right| \rightarrow 0 \text { as } t \rightarrow \infty, 0<\eta \leq r \leq 1
$$

for every $\eta>0$, which proves the theorem under (2.13).

We will now show that (2.13) holds. Again using the parabolic regularity of $u^{*}$ we have that $u_{r}^{*}(r, t)$ is bounded in $C^{a, a / 2}\left([\eta, 1] \times\left[t_{0}, \infty\right)\right)$, hence $\left|u_{r}^{*}(1, t)\right|$ is bounded as $t \rightarrow \infty$. This implies the existence of a subsequence of time, say $t_{n}$, such that

$$
u_{r}^{*}\left(1, t_{n}\right) \rightarrow a<0 \text { as } t_{n} \rightarrow \infty
$$

(As we are restricting $u_{0}$ to satisfy $u_{0} \geq w$ we know that $u^{*} \geq w$ and hence $u_{r}^{*}(1, t) \leq w^{\prime}(1)$.)

Considering now the initial value problem,

$$
\begin{gathered}
w_{a}^{\prime \prime}+\frac{N-1}{r} w_{a}^{\prime}+\lambda f\left(w_{a}\right)=0,0<r<1 \\
w_{a}(1)=0, w_{a}^{\prime}(1)=a<0
\end{gathered}
$$

and setting $u^{*}(r, t)=U(r, t)+w_{a}(r)$, it is easily seen, by repeating the above procedure that

$$
u^{*}\left(r, t_{n}\right) \rightarrow w_{a}(r), t_{n} \rightarrow \infty \text {, as } n \rightarrow \infty
$$

uniformly for $r$ in $[\eta, 1]$. Note that the initial value problem $(2.20)$ has at most one solution in $0<r<1$. We want to exclude the case of $a \neq w^{\prime}(1)$. Indeed, let us assume that $a \neq w^{\prime}(1)$. If $(2.20)$ had a bounded solution $w_{a}(r)$ in $(0,1)$, then this would contradict the uniqueness of the solution to (1.4) or (2.2) since $0<\lambda<\lambda_{\text {s. }}$ On the other hand, if $w_{a}(r)$ was unbounded with $w_{a}(r) \rightarrow+\infty$ as $r \rightarrow 0$ this would contradict the hypothesis $\left(H_{2}\right)$. There remains the case $w_{a}(r) \rightarrow-\infty$ as $r \rightarrow 0$. Taking now $\eta$ sufficiently small the last limit implies that $w_{a}(\eta)<0$ and from (2.21) we deduce that $u^{*}\left(\eta, t_{n}\right) \rightarrow w_{a}(\eta)$ as $t_{n} \rightarrow \infty$ or $u^{*}\left(\eta, t_{n}\right)<0$ for $t_{n}$ large enough. But the last statement contradicts the positivity of $u^{*}(r, t)$ on $(0,1)$ for all $t>0$. Hence $a=w^{\prime}(1)$ for all sequences $\left(t_{n}\right)$ so that $(2.13)$ holds. This completes the proof of the theorem.

We expect that the above results can be generalised to cover Robin boundary conditions, as well as the unforced case, i.e. $f(0)=0$. We conjecture that it is possible to get some sort of convergence even in the case when $\Omega$ is not a ball. 


\section{Blow-up for large initial data}

In this section we again consider $u^{*}=u\left(x, t ; u_{0}^{*}\right)$ to be a global classical unbounded solution, i.e. $\left(H_{1}\right)$ is satisfied and moreover the following condition holds:

$$
\Omega \text { is convex and } u_{0}^{*} \in C(\bar{\Omega}) \text {. }
$$

Our purpose is to show, under some growth restrictions on $f$, that taking $\hat{u}_{0}>u_{0}^{*}$, $\hat{u}=u\left(x, t ; \hat{u}_{0}\right)$ blows up in finite time. This manifests the critical nature of $u_{0}^{*}:$ for $\hat{u}_{0}>u_{0}^{*}$ in $\Omega, \hat{u}$ blows up in finite time, whereas for $u_{0}<u_{0}^{*} u\left(x, t ; u_{0}\right) \rightarrow w(x)$ uniformly as $t \rightarrow \infty$; also $u^{*}=u\left(x, t ; u_{0}^{*}\right) \rightarrow w(x)$ as $t \rightarrow \infty$ for any $x \in \Omega-\{0\}$, at least if $\Omega$ is a ball (see section 2). We assume $f$ is such that

$$
f(s)=k e^{a s}, s \geq 0 \text { and for } k, a>0 .
$$

Our main result is the following:

Theorem 3.1. Let $u^{*}$ be a solution of $(1.1)$, and hypothesis $\left(H_{1}\right)$ and conditions $(3.1,2)$ all hold. If $\hat{u}_{0}>u_{0}^{*}$ in $\Omega$, then the solution $\hat{u}=u\left(x, t ; \hat{u}_{0}\right)$ of $(1.1)$ blows up in finite time.

The proof will be given after some preliminary results. Throughout this section, we keep the convention that $u_{0}, u_{0}^{*}, \hat{u}_{0}$ always have the order

$$
u_{0}(x)<u_{0}^{*}(x)<\hat{u}_{0}(x), x \text { in } \Omega
$$

Hence by comparison we obtain $u<u^{*}<\hat{u}, x$ in $\Omega, t>0$ where $u, u^{*}, \hat{u}$ are solutions of (1.1) with initial data $u_{0}, u_{0}^{*}, \hat{u}_{0}$ respectively.

We now consider the following linearized problem about $u^{*}$ :

$$
\begin{gathered}
z_{t}=\Delta z+\lambda f^{\prime}\left(u^{*}\right) z, x \text { in } \Omega, t>0 \\
z=0, x \text { on } \partial \Omega, t>0 \\
z(x, 0)=z_{0}(x)>0, x \text { in } \Omega, t=0
\end{gathered}
$$

and we have:

Lemma 3.2. (a) If $u(x, 0)=u_{0}(x) \geq u_{0}^{*}-\gamma z_{0}$ then $u(x, t) \geq u^{*}(x, t)-\gamma z(x, t), x$ in $\Omega, t>0$.

(b) If $0<z_{0}(x) \leq \hat{u}_{0}(x)-u_{0}^{*}(x)$ then $\hat{u}(x, t) \geq u^{*}(x, t)+z(x, t), x$ in $\Omega t>0$.

Proof. (a) Setting $v=u^{*}-\gamma z$ we observe that,

$$
\frac{\partial v}{\partial t}-\Delta v-\lambda f(v)=\lambda \gamma\left(f^{\prime}(s)-f^{\prime}\left(u^{*}\right)\right) z<0
$$

since $u^{*}>s>u^{*}-\gamma z$ and $z>0$ in $\Omega(z>0$ on $(0, T] \times \Omega$, for any $T>0$ by the maximum- 
minimum principle). Also $v \leq u$ at $t=0$ and $v=u$ on the boundary $\partial \Omega$ for every $t>0$. Hence $v$ is a lower solution to (1.1) with $u(x, 0)=u_{0}(x)$ and $v \leq u$, in $\Omega, t \geq 0$.

(b) We now set $v(x, t)=u^{*}(x, t)+z(x, t)$ and have

$$
\frac{\partial v}{\partial t}-\Delta v-\lambda f(v)=\lambda\left(f^{\prime}\left(u^{*}\right)-f^{\prime}(s)\right) z<0
$$

since $z>0$ and $u^{*}+z>s>u^{*}$ in $\Omega$. Moreover $v \leq \hat{u}$ at $t=0$ and $v=\hat{u}$ on the boundary $\partial \Omega$. Hence $v$ is a lower solution to (1.1) with $u(x, 0)=\hat{u}_{0}$ and $v \leq \hat{u}$, which completes the proof of the lemma.

We consider now the linear eigenvalue problem,

$$
\begin{gathered}
\Delta \psi+\lambda f^{\prime}(w) \psi=\mu \psi, x \text { in } \Omega . \\
\psi=0, x \text { on } \partial \Omega .
\end{gathered}
$$

where $w$ is the unique solution to the steady state problem (1.4). It is known, $[1,7]$, that for the principal eigenpair $\left(\mu_{1}, \psi_{1}\right) \mu_{1}<0$, as a consequence of the minimality of $w$, $\psi_{1} \geq 0$, and $\psi_{1} \neq 0$. For $u_{0}<u_{0}^{*}, u$ is uniformly bounded, $u\left(x, t ; u_{0}\right) \rightarrow w(x)$ uniformly as $t \rightarrow \infty[12,13]$. Therefore for $t$ large, we have,

$$
w(x) \leq u(x, t) \leq w(x)+\varepsilon \psi_{1}(x) e^{\mu_{1} t}+O\left(e^{2 \mu_{1} t}\right)
$$

for some constant $\varepsilon>0$ ( $\varepsilon$ depending upon $u_{0}$ ) [7]. From Lemma 3.2(a) and (3.4) we obtain

$$
\gamma z \geq u^{*}-w-\varepsilon \psi_{1} e^{\mu_{1 t}}-O\left(e^{2 \mu_{1} t}\right)
$$

for $t$ large.

As the next step we have the proposition:

Proposition 3.3. Let $u^{*}=u\left(x, t ; u_{0}^{*}\right)$ be the solution of $(1.1)$, and $\left(H_{1}\right),(1.2,3)$ and $(3.1,2)$ be satisfied, then

$$
\int_{\mathbf{\Omega}} f^{q}\left(u^{*}\right) d x \rightarrow \infty \text { as } t \rightarrow \infty \text { for } q \geq N
$$

Proof. To obtain this we use a result of Friedman and McLeod [3]. Let $M(t)=$ sup $u^{*}(\cdot, t)$, then for $t$ large enough,

$$
\left|\nabla u^{*}(\cdot, t)\right|^{2} \leq 2 \int_{u^{*}(x, t)}^{M(t)} f(s) d s, x \text { in } \Omega, t \gg 0,
$$


(this uses $M(t) \rightarrow \infty$ as $t \rightarrow \infty)$. We define $x_{0}(t)$ by $u^{*}\left(x_{0}, t\right)=M(t)$ and introduce polar coordinates $(r, \theta)$ about $x_{0}(t) ;(3.6)$ implies

$$
\left|\frac{\partial u^{*}}{\partial r}\right|^{2} \leq 2 \int_{u^{*}}^{M} f(s) d s \leq 2\left(M-u^{*}\right) f(M)
$$

Integrating over the interval $(0, r)$, we find that

$$
u^{*}(x, t) \geq \frac{1}{2} M \text { for } r \leq M^{1 / 2} f^{-1 / 2}(M)=r_{0}
$$

Considering the ball $B\left(x_{0}, r_{0}\right)$, and using (3.7) we obtain

$$
\begin{aligned}
& \int_{\Omega} f^{q}\left(u^{*}\right) d x \geq f^{q}\left(\frac{M}{2}\right) \underset{B\left(x_{0}, r_{0}\right)}{ } d x=c f^{q}\left(\frac{M}{2}\right) \cdot r_{0}^{N} \\
& =c f^{q}\left(\frac{M}{2}\right) M^{N / 2} f^{-N / 2}(M) \geq c K e^{a(q-N) M / 2} M^{N / 2},
\end{aligned}
$$

where $M=M(t)$, and $c$ is a positive constant depending upon $N$. Now taking the limit in (3.8) as $t \rightarrow \infty$, provided $q \geq N$, the desired result follows.

Proof of the theorem. We take $\hat{u}_{0}>u_{0}^{*}$ in $\Omega$ and choose $u_{0}>0$ and $\gamma>0$ such that

$$
\frac{u_{0}^{*}-u_{0}}{\gamma} \leq \hat{u}_{0}-u_{0}^{*}=z_{0}
$$

From Lemma 3.2 and $(3.2,5)$ we obtain,

$$
\begin{gathered}
f(\hat{u})=k \exp (a \hat{u}) \geq k \exp \left[a\left(u^{*}+z\right)\right] \\
\geq k \exp \left[a\left(1+\frac{1}{\gamma}\right) u^{*}\right] \exp \left[-\frac{a}{\gamma}\left(w+\varepsilon \psi_{1}^{\mu_{1 t}}+O\left(e^{2 \mu_{1 t} t}\right)\right)\right] \\
\geq k B \exp \left[a\left(1+\frac{1}{\gamma}\right) u^{*}\right]
\end{gathered}
$$

where

$$
B=\exp \left[-\frac{a}{\gamma}\left(\|w\|+\varepsilon\left\|\psi_{1}\right\| e^{2 \mu_{1} t_{0}}+O\left(e^{2 \mu_{1} t_{0}}\right)\right)\right]
$$

$t \geq t_{0}>0$, for some $t_{0}>0$. 
We may choose now $\gamma$ such that

$$
1+\frac{1}{\gamma}=q \geq N, \text { or } \gamma=\frac{1}{q-1}, q \geq N
$$

Thus for these values of $\gamma,(3.9)$ and Proposition 3.3 imply:

$$
\int_{\Omega} f(\hat{u}) d x \geq B k \int_{\Omega} \exp \left(q a u^{*}\right) d x=B \int_{\mathbf{\Omega}} f^{q}\left(u^{*}\right) d x \rightarrow \infty \text { as } t \rightarrow \infty .
$$

Now we are ready to show that $\hat{u}$ blows up in finite time if $\hat{u}_{0}>u_{0}^{*}$. By introducing the functional

$$
a(t)=\int_{\mathbf{\Omega}} \hat{u}(x, t) \varphi_{1}(x) d x
$$

where $\left(\lambda_{1}, \varphi_{1}\right)$ is the principal eigenpair of $-\Delta$ with Dirichlet boundary conditions so $\lambda_{1}>0$ with $\varphi_{1}>0$ in $\Omega$ and taking $\left\|\varphi_{1}\right\|_{21}=1$, we obtain from (i.1),

$$
a^{\prime}(t)=\int_{\Omega} \hat{u}_{t} \varphi_{1} d x=-\lambda_{1} a(t)+\lambda \int_{\Omega} f(\hat{u}) \varphi_{1} d x
$$

Following $\mathrm{Ni}$ et al. [13], a result of Gidas et al. [5] may be applied for a convex region to give

$$
\int_{\mathbf{\Omega}} f(\hat{u}) d x \leq(m+1) \int_{\Omega_{0}} f(\hat{u}) d x, t \geq t_{0}
$$

where $\Omega_{0}$ is a subregion of $\Omega$, i.e. $\bar{\Omega}_{0} \subset \Omega$ and $m$ is some positive integer; this result uses the fact that $\hat{u}$ decreases near $\partial \Omega$. Now (3.11) yields:

$$
a^{\prime}(t) \geq-\lambda_{1} a+\lambda K \int_{\Omega_{0}} f(\hat{u}) d x
$$

where $K=\inf _{\Omega_{0}} \varphi_{1}>0$ (note that $\inf _{\Omega} \varphi_{1}=0$ ) for some $\bar{\Omega}_{0} \subset \Omega$. But from (3.10) we have $\int_{\Omega} f(\hat{u}) d x \rightarrow \infty$ as $t \rightarrow \infty$ so (3.12) implies

$$
\int_{\mathbf{\Omega}_{0}} f(\hat{u}) d x \rightarrow \infty \text { as } t \rightarrow \infty,
$$

and it follows from (3.13) that $a(t) \rightarrow \infty$ as $t \rightarrow \infty$.

Now applying Jensen's inequality to (3.11)

$$
a^{\prime}(t) \geq-\lambda_{1} a+\lambda f(a)
$$


Taking $T$ such that $\lambda f(s)-\lambda_{1} s>0$ for $s \geq a(T)$, and integrating (3.14), we get

$$
t-T \leq \int_{a(T)}^{a(1)} \frac{d s}{-\lambda_{1} s+\lambda f(s)} \leq \int_{a(T)}^{\infty} \frac{d s}{-\lambda_{1} s+\lambda f(s)}=c<\infty
$$

which implies that $a(t)$ becomes infinite in finite time $t^{*} \leq c+T$. Hence $\hat{u}$ blows up in finite time since $a(t) \leq \sup \hat{u}(\cdot, t)$.

Note that this proof is easily adapted to problems with Robin boundary conditions.

\section{Discussion}

In this paper we have discussed the implications of there being an unbounded, but classical, solution to (1.1) in cases where (1.4) has a unique classical and no singular weak solution $\left(\lambda<\lambda_{*}\right)$. In a previous paper such classical unbounded solutions have been found to exists, but, in that case, there was no classical steady state but instead a weak singular stationary solution $\left(\lambda=\lambda^{*}=\lambda_{\text {s }}\right)$. For this latter case any small pertubation of the initial data left the qualitative behaviour unchanged.

Here we have looked at problems with solutions $u^{*}$ given as the supremum (equivalently, the limit of a monotonic increasing sequence) of solutions $u$ which converge to the (unique) steady state as $t \rightarrow \infty$. It has already been found that such $u^{*}$ must be both unbounded and an $L^{1}$ solution of (1.1). It had already been established that for certain situations, e.g. $f(u)=e^{u}$ with $3 \leq N \leq 9, u^{*}$ could have this behaviour through blowing up partially, and consequently any increase of initial data results in complete blow-up. We have now examined the only alternative, namely that $u^{*}$ never blows up but instead satisfies $\sup u^{*}(\cdot, t) \rightarrow \infty$ as $t \rightarrow \infty$. We have seen (Section 2 ) that for radially symmetric problems $u^{*}(0, t) \rightarrow \infty$ as $t \rightarrow \infty$ whereas, for any $r>0, u^{*}(r, t) \rightarrow w(r)$. We conjecture that similar properties should hold for asymmetric problems: $u^{*}$ has a divergence set $D$ of measure zero and $u^{*} \rightarrow w$, pointwise, as $t \rightarrow \infty$ for $x \in \Omega \backslash D$. We have also found that for exponential nonlinearities with $\Omega$ being convex $u^{*}$ is again critical: not only does $u_{0}<u_{0}^{*}$ result in $u \rightarrow w$ as $t \rightarrow \infty$ but $\hat{u}_{0}>u_{0}^{*}$ implies that $\hat{u}$ blows up completely. We again conjecture that results like these should apply even for $\Omega$ not convex and for $f$ either growing faster than exponentially, or somewhat more slowly than exponentially (but still maintaining the requirement necessary for blow-up, (1.3)).

Knowledge of such $u^{*}$, whether they blow up partially or are instead classical but unbounded, has some practical significance. The simplest model for exothermic reactions is of the form (1.1) with $f(u)=e^{u}$. Then $u^{*}$ is critical in that it separates values of $u$ (i.e. of temperature) that have subcritical behaviour $-u<u^{*}$ means that $u \rightarrow w$ as $t \rightarrow \infty$ and no violent reactions occurs-from supercritical behaviour- $u>u^{*}$ means, at least if $\Omega$ is convex, that $u$ blows up and ignition takes place. Both critical phenomena do not appear to result in true physical blow-up. For partial blow-up temperature $u^{*}$ decays after the blow-up time and a (weak) solution to (1.1) continues for all time. For classical unbounded $u^{*}$ it only becomes large as $t$ gets large, so one can expect that effects such as reactant depletion with actually limit the size of $u$. 
Acknowledgement. The author is grateful for fruitful discussions with A. A. Lacey. It is hereby acknowledged the support from grant number ERBCHRXCT 930439 under the EC Human Capital and Mobility Scheme.

\section{REFERENCES}

1. H. AMANN, Fixed point equations and nonlinear eigenvalue problems in ordered Banach spaces SIAM Rev. 18 (1976), 620-709.

2. J. Bebernes and D. Eberly, Mathematical Problems from Combustion Theory (SpringerVerlag, New York, 1989).

3. A. Friedman and B. MCLeod, Blow-up of positive solutions of semilinear heat equations, Indiana Univ. Math. J. 34 (1985), 425-447.

4. H. Fujita, On the nonlinear equations $\Delta u+e^{u}=0$ and $u_{t}=\Delta u+e^{u}$, Bull. Amer. Math. Soc. 75 (1969), 132-135.

5. B. Gidas, W.-M. Ni and L. Nirenberg, Symmetry and related properties via the maximum principle, Comm. Math. Phys. 68 (1979), 209-243.

6. D. D. Joseph and T. S. Lundgren, Quasilinear Dirichlet problems driven by positive sources, Arch. Rational Mech. Anal. 49 (1973), 241-269.

7. H. B. Keller and D. S. Cohen, Some positive problems suggested by nonlinear heat generation, J. Math. Mech. 16 (1967), 1361-1376.

8. A. A. LACEY, Mathematical analysis of thermal runaway for spatially inhomogeneous reactions, SIAM J. Appl. Math. 43 (1983), 1350-1366.

9. A. A. LACEY and D. Tzanetis, Global existence and convergence to a singular steady state for a semilinear heat equation, Proc. Roy. Soc. Edinburgh Sect. A 105 (1987), 289-305.

10. A. A. LaCEy and D. E. Tzanetis, Global, unbounded solutions to a parabolic equation, $J$. Differential Equations 101 (1993), 80-102.

11. O. A. Ladyzhenskaja, V. A. Solonnikov and N. N. Uralceva, Linear and quasilinear equations of parabolic type, in Trans. Math. Monographs 23 (Amer. Math. Soc., Providence, RI, 1968).

12. H. Matano, Asymptotic behavior and stability of solutions of semilinear diffusion equations, Publ. Res. Inst. Math. Sci. 15 (1979), 401-454.

13. W.-M. Ni, P. E. Sacks and J. Tavantzis, On the asymptotic behavior of solutions of certain quasilinear parabolic equations, J. Differential Equations 54 (1984), 97-120.

14. W.-M. Ni and P. E. SACKs, The number of peaks of positive solutions of semilinear parabolic equations, SIAM J. Math. Anal. 16 (1985), 460-471.

15. W.-M. $\mathrm{N}_{1}$ and P. E. SACKS, The singular behavior in nonlinear parabolic equations, Trans. Amer. Math. Soc. 287 (1985), 657-671.

16. D. H. SatTinger, Monotone methods in nonlinear elliptic and parabolic boundary value problems, Indiana Univ. Math. J. 21 (1972), 979-1000.

Department of Mathematics

National Technical University

Zografou Campus

15780 ATHENS

Greece 